\section{PTH-188 SMALL BOWEL DIAPHRAGM DISEASE: DIAGNOSIS WITH CAPSULE ENDOSCOPY AND TREATMENT WITH BUDESONIDE}

doi:10.1136/gutjnl-2013-304907.675

1."R Pandey, 'M Khan, 'N Van Someren, 'K Besherdas. 'Gastroenterology, Barnet \& Chase Farm NHS Trust, London, UK

Introduction Diaphragm disease is characterised by strictures that mainly occur in the small intestine, resulting from the use of nonsteroidal anti-inflammatory drugs. It may present with vague gastrointestinal symptoms or as a surgical emergency requiring laparotomy. Most cases are diagnosed retrospectively at laparotomy. Laparoscopy and various radiological imaging modalities are often inconclusive. Capsule endoscopy effectively diagnoses diaphragm disease pre-operatively. Following withdrawal of the offending NSAID, surgical resection, strictureplasty and double balloon enteroscopy have all been used as potential treatments. Specific drug treatment of small bowel diaphragm disease has not been described. We present a case series of three patients who were diagnosed with Diaphragm disease at capsule endoscopy and responded to Budesonide therapy.

Methods Case notes of three patients with a confirmed diagnosis of Diaphragm Disease were analysed, with respect to presenting symptoms, duration of symptoms, investigations, treatment and follow-up.

Results Three female patients between the ages of 56 and 72 presented with gastrointestinal symptoms, including abdominal pain, nausea, vomiting, bloating and loose stool. Symptoms were present from a range of two months to four years before their presentation to secondary care. All patients had a history of regular NSAID use and were investigated with gastroscopy, colonoscopy and CT abdomen, all of which were normal. Small bowel MRI was also normal in two patients (Fig.1). Capsule endoscopy in all patients demonstrated characteristic features of diaphragm disease (Fig.2). All patients received a tapering dose of Budesonide, starting at a dose of $9 \mathrm{mg}$. A symptomatic response was observed in all patients. One patient has now been started on Azathioprine to maintain long term remission. Two remain on reducing doses of steroid with a significant improvement in symptoms.

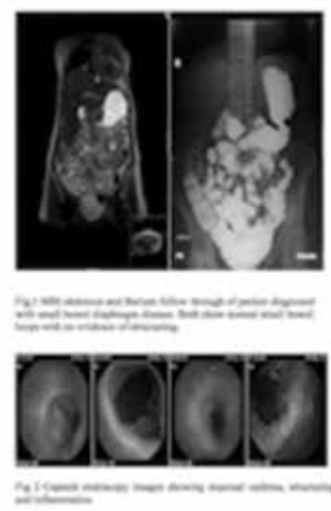

Abstract PTH-188 Figure 1

Conclusion Diaphragm disease is an under recognised clinical entity that can present in a variety of ways. It can be difficult to diagnose with routine endoscopy or conventional radiology. Capsule endoscopy appears to be the most sensitive test. Clinicians should have a high index of suspicion for this condition, particularly in the context of NSAID use and chronic gastrointestinal symptoms. Budesonide appears to be an effective therapy for Diaphragm Disease, and it seems likely that long-term therapy with azathioprine can maintain remission. However this is an observational study with a small num- ber of patients. Further research is required to validate this as successful, viable, evidence based treatment option.

Disclosure of Interest None Declared.

\section{PTH-189 DOUBLE BALLOON ENTEROSCOPY IN THE ELDERLY WITH OBSCURE GI BLEEDING- IS IT WORTH THE PUSH AND PULL?}

doi:10.1136/gutjnl-2013-304907.676

1.* R Sidhu, 1,2S Hardcastle, 'D S Sanders. 'Gastroenterology; ${ }^{2}$ Royal Hallamshire Hospital, Sheffield, UK

Introduction Double Balloon enteroscopy (DBE) is an increasingly important procedure particularly in the context of obscure GI bleeding (OGB) with a high reported diagnostic yield. There is however paucity of data on its use in the elderly. The aim of this study was to assess the utility of $\mathrm{DBE}$ in the elderly and risk of complications compared to a younger cohort.

Methods A prospective review of consecutive patients who underwent DBE over the last six years for the indication of OGB was conducted. The majority of patients underwent a capsule endoscopy prior to DBE, either locally or at our centre. Data was collected on demographics, procedure duration, dose of sedation/analgesia, diagnostic yield and subsequent change in management and complications encountered. Patients were divided into group 1: age $\geq 70$ years, group 2: age $<70$ years.

Results One hundred and forty eight DBE procedures were carried out for the indications of OGB. The majority were for the indication of iron deficiency anaemia (IDA, $\mathrm{n}=109.74 \%$ ), 53\% were males and the oral route was carried out in $69 \%$ of patients. Group 1 (age $\geq 70$ years) consisted of $27 \%(n=40)$ of the cohort with a mean age of 77 years (range $70-83$ years). There were $38 \%$ of patients with the presence of significant co-morbidity and four patients were on warfarin. The diagnostic yield in Group 1 was 53\%. There was no significant difference in the yield between those with IDA and overt bleeding $(p=0.7)$. The commonest diagnosis in the elderly was angioectasia $(45 \%, \mathrm{n}=18)$. The other findings included a small bowel tumour and mantle cell lymphoma $(\mathrm{n}=2)$ and a Meckels diverticulum $(n=1)$. Therapeutics was performed in $45 \%$. There was one respiratory arrest in a patient with known chronic airways disease (COPD). This patient was subsequently discharged home after a short stay on intensive care. The mean age in group $2(n=108)$ was 54 years, $44 \%$ females and the diagnostic yield was $35 \%$. Comparison of the two groups demonstrated a trend towards a higher diagnostic yield in the elderly $(p=0.06)$. On logistic regression, previous transfusion requirement was associated with a higher yield with DBE in all patients $(p=0.04)$. Subsequent management was altered in a significant greater proportion of the elderly $(28 \%$ versus $50 \%, p=0.01$, OR $2.6,95 \%$ CI 1.2-5.5). There was no difference in the duration of the procedure between the two groups $(p=0.4)$, whilst the median dose of midazolam and fentanyl was significantly greater in group 2 (4.5 mg versus $6 \mathrm{mg}, \mathrm{p}<0.001,50 \mathrm{mcg}$ versus $75 \mathrm{mcg}$, $\mathrm{p}<0.001$ respectively). There were no complications in group 2 .

Conclusion DBE has a high diagnostic yield with a positive impact on patient management in the elderly. Albeit low rate of complications, careful selection of patients would help reduce risks in this age group.

Disclosure of Interest None Declared.

\section{PTH-190 DOES THE AMOUNT OF SEDATION HAVE AN IMPACT ON THE DIAGNOSTIC YIELD OF DOUBLE BALLOON ENTEROSCOPY ? EXPERIENCE FROM A TERTIARY CENTRE}

doi:10.1136/gutjnl-2013-304907.677

1.*R Sidhu, 'S Hardcastle, 'D S Sanders. 'Gastroenterology, Royal Hallamshire Hospital, Sheffield, UK 
Introduction Double balloon enteroscopy (DBE) offers the ability for diagnostic and therapeutic intervention in the small bowel. The procedure takes an average of an hour to carry out and can be uncomfortable for the patient. It also has an associated learning curve for the endoscopist. The depth of insertion is a subjective estimation of the total number of passes into the small bowel. The success/diagnostic yield of the procedure relies upon patient tolerability and locating the target lesion or bleeding point. The aim of this study was to assess the technical success rate for routine DBE over time in comparison to sedation dose used.

Methods A prospective review of the $290 \mathrm{DBE}$ procedures done since the start of the service was conducted. The majority of patients underwent a capsule endoscopy either locally or at our centre prior to DBE which helped to guide the chosen route. Data was collected for sedation/analgesia used, procedure length, number of passes into the small bowel and diagnostic yield between the initial 145 (group 1) and latter 145 procedures (group 2). Similar comparisons were also done between the oral and anal routes of DBE.

Results The DBE procedures were performed from July 2006 to Nov 2012 by two endoscopists. Whilst the median doses of midazolam used between the two groups were similar (median $5 \mathrm{mg}$ versus $6 \mathrm{mg}, \mathrm{p}=0.8$ ), a greater amount of fentanyl was used in group 2 (median $50 \mathrm{mcg}$ versus $100 \mathrm{mcg}, \mathrm{p}<0.001$ ). There was no difference in the procedure length or the number of passes recorded by the endoscopists. However there was a significant increase in the diagnostic yield in the latter group (32\% versus $58 \%, \mathrm{p}<0.001)$.

A total of 165 oral DBE procedures were done. Whilst there were no differences in the procedures length or number of passes into the small bowel, a greater amount of fentanyl was used in the latter half of the procedures. The diagnostic yield improved significantly in the latter half of the oral DBE procedures ( $41 \%$ versus $61 \%, p=0.01$ ). There were 125 anal DBE procedures. There was no difference in procedure characteristics or diagnostic yield for the anal route over time despite higher doses of fentanyl.

Conclusion This study demonstrates an improved diagnostic yield for DBE over time particularly with the oral route and with a greater amount of fentanyl used. The absence of improvement in yield for the anal route in this study is consistent with the literature to date. The anal route remains a challenge for endoscopists due to difficulty achieving a stable platform in the terminal ileum to progress. More education on retrograde techniques would help overcome this limitation.

Disclosure of Interest None Declared.

\section{PTH-191 CASE SERIES OF 162 VIDEO CAPSULE ENDOSCOPIES FROM A SINGLE UK TERTIARY REFERRAL CENTRE}

doi:10.1136/gutjnl-2013-304907.678

1,"R A Ley Greaves, 'E Russo, ' 'J Faulkner, 'P Gummett, 'J Hoare. 'Gastroenterolgoy, Imperial College, London, UK

Introduction Video capsule endoscopy (VCE) is now a routine procedure for investigating the small bowel. It is not apparent whether the yield and utility of the procedure is the same when used in routine practise, compared to earlier studies ${ }^{1-3}$. The yield of the procedure is important when planning local provision of enteroscopy services.

Methods We retrospectively audited 162 consecutive procedures in a single UK centre. In our analysis we defined diagnostic yield as confirming or establishing a diagnosis or definitively refuting a diagnosis previously suggested by other investigations. Patients were fasted for at least 8 hours and not given bowel preparation. Equipment was from Olympus UK. Studies were initially read by a nonmedically qualified healthcare worker before approval by the senior author, after a learning phase when the initial 50 examinations were dual read. Patency capsules were used in patients with obstructive type symptoms or known Crohn's disease.
Results The overall diagnostic yield was $35 \%$ which is slightly lower but comparable to early studies ${ }^{3}$. The yield for overt GI bleeding (OGIB) was $43 \%$, lower than the often reported yield of approximately $60 \%$. Of the 162 patients 14 were referred on for enteroscopy (8.6\%) which is lower than many early reports. Of the 14 referred, 10 were for anaemia and 4 for OGIB. 11 had successful therapy with balloon enteroscopy and 1 was diagnosed and a tattoo placed for lesion resection. In 9/162 studies (5.5\%) pathology was found within reach of gastroscopy or colonoscopy and had been missed on previous examination. One retained capsule required surgical removal $(0.6 \%)$. Four capsules were retained in the stomach $(2.4 \%)$ and 30 were seen to enter the small bowel but did not reach the caecum $(18.5 \%) .16$ studies were deemed inadequate due to food debris (9.8\%). 2 failed due to equipment malfunction. Total incomplete examination rate was therefore $30 \%$. Average small bowel transit time was 281 minutes (range 90-710). Anaemia $(n=87)$ was the most common indication with a diagnostic yield of $33 \%$. OGIB $(n=37)$ was the next most common indication yielding $43 \%$. Query Crohn's ( $n=22$ ) had a yield of $14 \%$. Malabsorption query Coeliac $(n=5)$ yielded $40 \%$. Unsurprisingly known Coeliac $(n=2)$ gave a complete yield of $100 \%$. Abnormal Imaging $(n=4)$ returned a $75 \%$ yield. Weight loss $(n=1)$ gave $0 \%$ yield. Abdo pain $(n=4)$ had a $25 \%$ yield.

Conclusion In routine practise VCE is a useful technique to diagnose patients with small bowel disorders. Yield seems slightly lower than previous studies but is still significant. Incomplete examination rate was high, mostly due to inadequate battery life. Only $8.6 \%$ of patients required enteroscopy which has implications for service provision.

\section{Disclosure of Interest None Declared.}

\section{REFERENCES}

1. Triester et al. 2005

2. Marmo et al. 2005

3. Hartmann et al.2005

\section{PTH-192 ONCE NEGATIVE ALWAYS NEGATIVE? - THE CLINICAL UTILITY OF REPEATING TTG}

doi:10.1136/gutjnl-2013-304907.679

1."S Merson, 'K So, 'R Brown, 'T Ahmad. 'Royal Devon and Exeter Hospital, Exeter, UK

Introduction Patients with chronic or recurrent abdominal symptoms, or anaemia, often undergo repeated rounds of investigations including tissue transglutaminase (TTG) antibody testing. The aim of this study was to investigate a) the number of patients having multiple TTG tests $b$ ) the risk of having a positive TTG after a past negative test.

Methods A list of all TTG requests between 01/01/02-15/07/12 at our laboratory was created. Patients with an initial negative result, but subsequent positive result, were identified, and their hospital and GP records reviewed. Our institution employed the Aesku (Grifols) test prior to 2007 and then the Orgentec test (Launch Diagnostics) and has not used these tests to monitor patients with known Coeliac disease.

Results 44,985 unique patients (65.8\%female, 13.5\% under 18 years old) had at least one TTG test carried out over the 10.5-year study period. Use of the TTG test has increased (4 new patient tests in 2002 vs. 11,466 in 2011). $2.0 \%$ of first TTG tests were positive (2.2\% of the total children and $2.0 \%$ of the total adults).5,872 patients $(69.6 \%$ female, $16.6 \%$ of the total children and $12.5 \%$ of the total adults) had more than 1 test, of which 4,813patients $(10.7 \%)$ had 2 tests and $1059(2.3 \%)$ had 3-10 tests. The median interval between the first and the last test was 21 months (range 1 day - 12.6 yrs). Of the patients with more than one test 17 (7 children), without a prior diagnosis of coeliac disease (CD), had a negative TTG test (whilst taking a normal diet) followed by a positive test. These 